\title{
Blow-up of solutions for a nonlinear Petrovsky type equation with initial data at arbitrary high energy level
}

\section{Lishan Liu ${ }^{1,2}$, Fenglong Sun ${ }^{1 *}$ (D) and Yonghong $\mathrm{Wu}^{2}$}

${ }^{*}$ Correspondence:

sfenglong@sina.com

${ }^{1}$ School of Mathematical Sciences,

Qufu Normal University, Qufu,

People's Republic of China

Full list of author information is

available at the end of the article

\begin{abstract}
In this paper, we study the initial boundary value problem for a Petrovsky type equation with a memory term, nonlinear weak damping, and a superlinear source:

$$
u_{t t}+\Delta^{2} u-\int_{0}^{t} g(t-\tau) \Delta^{2} u(\tau) \mathrm{d} \tau+\left|u_{t}\right|^{m-2} u_{t}=|u|^{p-2} u_{,} \quad \text { in } \Omega \times(0, T) .
$$

When the source is stronger than dissipations, we obtain the existence of certain weak solutions which blow up in finite time with initial energy $E(0)=R$ for any given $R \geq 0$.
\end{abstract}

MSC: $35 \mathrm{~L} 70 ; 65 \mathrm{M} 60$

Keywords: Petrovsky type equation; Memory term; Nonlinear damping; Blow-up

\section{Introduction}

In this paper, we are concerned with the following initial boundary value problem for a nonlinear Petrovsky type equation with a memory term and nonlinear damping:

$$
\left\{\begin{array}{l}
u_{t t}+\Delta^{2} u-\int_{0}^{t} g(t-\tau) \Delta^{2} u(\tau) \mathrm{d} \tau+\left|u_{t}\right|^{m-2} u_{t}=|u|^{p-2} u, \quad \text { in } \Omega \times(0, T) \\
u(x, t)=\partial_{\nu} u(x, t)=0, \quad \text { on } \partial \Omega \times[0,+\infty), \\
u(x, 0)=u_{0}(x), \quad u_{t}(x, 0)=u_{1}(x), \quad \text { in } \Omega
\end{array}\right.
$$

where $m \geq 2, p>2, \Omega$ is a bounded domain of $\mathbb{R}^{n}(n \geq 1)$ with a smooth boundary $\partial \Omega$, $\partial_{\nu} u$ is the outer normal derivative of $u$, the relaxation function $g: \mathbb{R}^{+} \rightarrow \mathbb{R}^{+}$is nonincreasing with $\int_{0}^{\infty} g(s) \mathrm{d} s<1$, the initial data $\left(u_{0}, u_{1}\right) \in H_{0}^{2}(\Omega) \times L^{2}(\Omega)$. The Petrovsky type of equation

$$
u_{t t}+\Delta^{2} u=g\left(x, t, u, u_{t}\right)
$$

originated from the study of beams and plates, and it can also be used in many branches of physics such as nuclear physics, optics, geophysics, and ocean acoustics. In the past

(c) The Author(s) 2019. This article is distributed under the terms of the Creative Commons Attribution 4.0 International License (http://creativecommons.org/licenses/by/4.0/), which permits unrestricted use, distribution, and reproduction in any medium, provided you give appropriate credit to the original author(s) and the source, provide a link to the Creative Commons license, and indicate if changes were made. 
decades, a great deal of mathematical effort has been devoted to the study of wellposedness, regularity, asymptotic behavior for such kind of fourth order wave equations, as reported in [1-9] and the references therein.

In [10], Messaoudi studied the following nonlinearly damped semilinear Petrovsky equation:

$$
\left\{\begin{array}{l}
u_{t t}+\Delta^{2} u+a\left|u_{t}\right|^{m-2} u_{t}=b|u|^{p-2} u, \quad \text { in } \Omega \times(0, T) \\
u(x, t)=\partial_{\nu} u(x, t)=0, \quad \text { on } \partial \Omega \times[0,+\infty) \\
u(x, 0)=u_{0}(x), \quad u_{t}(x, 0)=u_{1}(x), \quad \text { in } \Omega
\end{array}\right.
$$

where $a, b>0$. It is showed that the solution blows up in finite time if $p>m$ and the initial energy is negative, and it globally exists if $p<m$. In [11], Messaoudi considered a nonlinear wave equation under the influence of a linear memory term and a nonlinear damping

$$
\left\{\begin{array}{l}
u_{t t}-\Delta u+\int_{0}^{t} g(t-\tau) \Delta u(\tau) \mathrm{d} \tau+\left|u_{t}\right|^{m-1} u_{t}=|u|^{p-1} u, \quad \text { in } \Omega \times(0, T), \\
u(x, t)=0, \quad \text { on } \partial \Omega \times[0,+\infty), \\
u(x, 0)=u_{0}(x), \quad u_{t}(x, 0)=u_{1}(x), \quad \text { in } \Omega
\end{array}\right.
$$

By using the method in [12], the author proved that the solution is global if $p \leq m$ and blows up in finite time with negative initial energy if $p>m$. In [13], Messaoudi improved the blow-up result in [11] to solutions with positive bounded initial energy. In [14], Chen and Zhou studied problem (1.2) and obtained the finite time blow-up results provided that the initial energy is positive bounded or vanishing. If the solution $u$ of problem (1.2) blows up in finite time, Zhou [5] presented an estimate for the lower bound of blow-up time. Following the strategy similar to that in [13], Li and Gao [15] studied problem (1.1). Under the following assumptions:

(a1) $p>m \geq 2$,

(a2) $\int_{0}^{\infty} g(s) \mathrm{d} s<1-\frac{1}{(p-1)^{2}}$,

(a3) $E(0)<E_{1}$ and $\left\|\Delta u_{0}\right\|_{L^{2}}>C$, where $E_{1}$ and $C$ are positive constants,

$\mathrm{Li}$ and Gao proved that the solution of problem (1.1) blows up in finite time.

In recent years, some researchers obtained the finite time blow-up results for different kinds of evolution equations with initial data at arbitrary high energy level. In [16], Todorova and Vitillaro studied the Cauchy problem for equation (1.3) without the memory term. The authors proved that, for any given $\alpha>0, \lambda \geq 0$, there exist infinitely many data $u_{0}, u_{1}$ in the energy space such that the initial energy $E(0)=\lambda$, the gradient norm $\left\|\nabla u_{0}\right\|_{L^{2}}=\alpha$, and the solution of the Cauchy problem blows up in finite time. By establishing a special second order differential inequality, Wang [4] studied the following initial boundary value problem of the fourth order wave equation with the nonlinear strain and source terms:

$$
\left\{\begin{array}{l}
u_{t t}+u_{t}+\Delta^{2} u-\alpha \Delta u-\sum_{i=1}^{n} \frac{\partial}{\partial x_{i}}\left(\theta_{i}(x)\right)=f(u), \quad \text { in } \Omega \times(0, T), \\
u(x, t)=\partial_{\nu} u(x, t)=0, \quad \text { on } \partial \Omega \times[0,+\infty), \\
u(x, 0)=u_{0}(x), \quad u_{t}(x, 0)=u_{1}(x), \quad \text { in } \Omega,
\end{array}\right.
$$

and established the finite time blow-up results of solutions with arbitrary initial energy, $-\infty<E(0)<+\infty$. In [17], Ye considered the following initial boundary value problem of 
higher order nonlinear viscoelastic wave equation:

$$
\left\{\begin{array}{l}
u_{t t}+(-\Delta)^{m} u-\int_{0}^{t} g(t-s)(-\Delta)^{m} u(s) \mathrm{d} \tau=|u|^{p-2} u, \quad \text { in } \Omega \times \mathbb{R}^{+}, \\
u(x, 0)=u_{0}(x), \quad u_{t}(x, 0)=u_{1}(x), \quad \text { in } \Omega, \\
\left.\frac{\partial_{i} u}{\partial v_{i}}\right|_{\partial \Omega}=0, \quad i=0,1,2, \ldots, m-1,
\end{array}\right.
$$

where $m \geq 1, p>2$. By using a special differential inequality, the author obtained the existence of certain solutions with nonpositive, positive bounded, or arbitrary high initial energy that blow up in finite time and also derived the life span estimates of these solutions. In [18], Song and Xue studied an equation similar to (1.3) except for the nonlinear weak damping term which is replaced by a linear strong damping term $-\Delta u_{t}$. By applying the technique similar to that in [18], Song [19] considered problem (1.3) and obtained the finite time blow-up result for certain solutions whose initial data have arbitrary high initial energy. By combining Song's method and the concavity method, we studied some parabolic type equations and hyperbolic equations with damping terms. We also obtained the finite time blow-up result for certain solutions whose initial data have arbitrary high initial energy, see [20-23]. For details of the study of other kinds of evolution equations, we refer the reader to [24-35].

To the best of our knowledge, there are no results about the finite time blow-up for solutions of problem (1.1) with arbitrary high initial energy. In this paper, following the strategy similar to that in [19] with minor modifications and under suitable conditions on $m, p$ and $g$ :

(a1) $p>m \geq 2$,

(a2) $\int_{0}^{\infty} g(s) \mathrm{d} s<1-\frac{1}{(p-1)^{2}}$

(a3') $0<E(0)<C \int_{\Omega} u_{0} u_{1} \mathrm{~d} x$, where $C$ is a positive constant,

we prove that there exist solutions of problem (1.1) that blow up in finite time. Moreover, we prove that, for any given $R>0$, there exists $\left(u_{0}, u_{1}\right) \in H_{0}^{2}(\Omega) \times L^{2}(\Omega)$ such that $E(0)=R$ and $\left(u_{0}, u_{1}\right)$ satisfies assumption (a3'). We finally obtain a set $\mathbb{B}_{1} \cup \mathbb{B}_{2}$ in the space $H_{0}^{2}(\Omega) \times$ $L^{2}(\Omega)$ such that the solution $u$ of problem (1.1) blows up in finite time provided that the initial data $\left(u_{0}, u_{1}\right) \in \mathbb{B}_{1} \cup \mathbb{B}_{2}$. Hence, we have extended the result of [15].

The rest of the paper is organized as follows. In Sect. 2, we present some notations and definitions and give the local existence theorem and some important lemmas. In Sect. 3, we prove our main results.

\section{Preliminaries}

Throughout this paper, we denote $\mathbb{R}^{+}=[0,+\infty)$ and the standard $L^{s}(\Omega)$-norm by $|u|_{s}=$ $\|u\|_{L^{s}(\Omega)}$. In view of the Sobolev-Poincaré inequality, the norm $\|w\|:=|\Delta w|_{2}$ is equivalent to the usual norm

$$
\|w\|_{H^{2}}=\left(|\Delta w|_{2}^{2}+|\nabla w|_{2}^{2}+|w|_{2}^{2}\right)^{\frac{1}{2}}
$$

on the Sobolev space

$$
H_{0}^{2}(\Omega)=\left\{w \in H^{2}(\Omega): w=\partial_{v} w=0 \text { on } \partial \Omega\right\} .
$$

We assume that $p, m$ and the relaxation function $g$ satisfy the following assumptions. 
(H1) $2<p<\infty$, if $n \leq 4 ; 2<p \leq \frac{2(n-2)}{n-4}$, if $n \geq 5$.

(H2) $2 \leq m<\infty$, if $n \leq 4 ; 2 \leq m \leq \frac{2 n}{n-4}$, if $n \geq 5$.

(H3) $g:[0,+\infty) \rightarrow[0,+\infty)$ is a $C^{1}$ non-increasing function satisfying

$$
l:=1-\int_{0}^{\infty} g(s) \mathrm{d} s>0 .
$$

Lemma 2.1 Suppose that (H1) holds. Then there exists a sequence of functions $\left\{v_{k}\right\} \in$ $H_{0}^{2}(\Omega)$ such that

$$
\frac{1}{2} \int_{\Omega}\left|\Delta v_{k}\right|^{2} \mathrm{~d} x+\frac{1}{2} \int_{\Omega}\left|v_{k}\right|^{2} \mathrm{~d} x-\frac{1}{p} \int_{\Omega}\left|v_{k}\right|^{p} \mathrm{~d} x \rightarrow+\infty \quad \text { as } k \rightarrow \infty .
$$

Proof It is easy to verify that the $C^{2}$ functional

$$
J(v):=\frac{1}{2} \int_{\Omega}|\Delta v(x)|^{2} \mathrm{~d} x+\frac{1}{2} \int_{\Omega}|v(x)|^{2} \mathrm{~d} x-\frac{1}{p} \int_{\Omega}|v(x)|^{p} \mathrm{~d} x, \quad v \in H_{0}^{2}(\Omega)
$$

is the Euler-Lagrange functional for the following boundary value problem:

$$
\left\{\begin{array}{l}
\Delta^{2} v+v=|v|^{p-2} v, \quad \text { in } \Omega, \\
v=\frac{\partial v}{\partial v}=0, \quad \text { on } \partial \Omega .
\end{array}\right.
$$

Similar to the proof of Theorem 3.7 in [36], by using the fountain theorem, we can prove that problem (2.1) has a sequence of weak solutions $\left\{v_{k}\right\} \subset H_{0}^{2}(\Omega)$ such that $J\left(v_{k}\right) \rightarrow+\infty$ as $k \rightarrow \infty$.

Similar to Definition 1.2 in $[37,38]$, we define the weak solution of $(1.1)$ as follows.

Definition 2.2 A function $u=u(x, t)$ is said to be a weak solution of (1.1) defined on the time interval $[0, T]$ for some $T>0$ provided that $u \in C\left([0, T] ; H_{0}^{2}(\Omega)\right)$ is such that $u_{t} \in$ $C\left([0, T] ; L^{2}(\Omega)\right) \cap L^{m}(\Omega \times(0, T))$ and

(i) $u(x, 0)=u_{0}(x), u_{t}(x, 0)=u_{1}(x)$;

(ii) The following variational identity holds for all $t \in[0, T]$ and all test functions $\phi \in \mathcal{F}$ :

$$
\begin{aligned}
& \int_{\Omega} u_{t}(t) \phi(t) \mathrm{d} x-\int_{\Omega} u_{t}(0) \phi(0) \mathrm{d} x-\int_{0}^{t} \int_{\Omega} u_{t}(\tau) \phi_{t}(\tau) \mathrm{d} x \mathrm{~d} \tau \\
& \quad+\int_{0}^{t} \int_{\Omega} \Delta u(\tau) \Delta \phi(\tau) \mathrm{d} x \mathrm{~d} \tau-\int_{0}^{t} \int_{0}^{s} g(s-\tau) \int_{\Omega} \Delta u(\tau) \Delta \phi(s) \mathrm{d} x \mathrm{~d} \tau \mathrm{d} s \\
& \quad+\int_{0}^{t} \int_{\Omega}\left|u_{t}(\tau)\right|^{m-2} u_{t}(\tau) \phi(\tau) \mathrm{d} x \mathrm{~d} \tau \\
& =\int_{0}^{t} \int_{\Omega}|u(\tau)|^{p-2} u(\tau) \phi(\tau) \mathrm{d} x \mathrm{~d} \tau,
\end{aligned}
$$

where

$$
\mathcal{F}=\left\{\phi: \phi \in C\left([0, T] ; H_{0}^{2}(\Omega)\right) \text { with } \phi_{t} \in C\left([0, T] ; L^{2}(\Omega)\right)\right\}
$$


Suppose that $u$ is a weak solution of (1.1), then we define the energy functional $E(t)$ by

$$
E(t)=\frac{1}{2}\left|u_{t}\right|_{2}^{2}+\frac{1}{2}(1-G(t))|\Delta u|_{2}^{2}+\frac{1}{2}(g \circ \Delta u)(t)-\frac{1}{p}|u|_{p}^{p},
$$

where $G(t):=\int_{0}^{t} g(s) \mathrm{d} s$ and

$$
(g \circ \Delta u)(t):=\int_{0}^{t} g(t-\tau)|\Delta u(t)-\Delta u(\tau)|_{2}^{2} \mathrm{~d} \tau
$$

We state the local existence theorem as follows.

Theorem 2.3 Assume that $m, p$, and $g$ satisfy $(\mathrm{H} 1)-(\mathrm{H} 3)$. Then, for any given $\left(u_{0}, u_{1}\right) \in$ $H_{0}^{2}(\Omega) \times L^{2}(\Omega)$, problem (1.1) has a unique weak solution $u$ such that

$$
u \in C\left([0, T] ; H_{0}^{2}(\Omega)\right), \quad u_{t} \in C\left([0, T] ; L^{2}(\Omega)\right) \cap L^{m}(\Omega \times(0, T))
$$

for some $T>0$. Moreover, we have the following energy identity:

$$
\begin{gathered}
E(t)-\frac{1}{2} \int_{0}^{t}\left(g^{\prime} \circ \Delta u\right)(\tau) \mathrm{d} \tau+\frac{1}{2} \int_{0}^{t} G(\tau)|\Delta u(\tau)|_{2}^{2} \mathrm{~d} \tau \\
+\int_{0}^{t}\left|u_{t}(\tau)\right|_{m}^{m} \mathrm{~d} \tau=E(0) \quad \text { for all } t \in\left[0, T^{*}\right),
\end{gathered}
$$

where $T^{*}$ is the maximal existence time for the weak solution $u$.

Proof Since $p$ satisfies (H1), the embedding $H_{0}^{2}(\Omega) \hookrightarrow L^{2(p-1)}(\Omega)$ is continuous and it holds that $|u|^{p-2} u \in L^{2}(\Omega)$ provided $u \in H_{0}^{2}(\Omega)$. We claim that the Nemytskii operator $f: u \mapsto|u|^{p-2} u$ is locally Lipschitz continuous from $H_{0}^{2}(\Omega)$ into $L^{2}(\Omega)$. In fact, for any $u, v \in H_{0}^{2}(\Omega)$, we have

$$
\begin{aligned}
|f(u)-f(v)|_{2} & \\
= & {\left[\int_{\Omega}\left(|u|^{p-2} u-|v|^{p-2} v\right)^{2} \mathrm{~d} x\right]^{\frac{1}{2}} } \\
\leq & (p-1)\left[\int_{\Omega}|\xi|^{2(p-2)}|u-v|^{2} \mathrm{~d} x\right]^{\frac{1}{2}} \\
\leq & (p-1)\left[\int_{\Omega}|u|^{2(p-2)}|u-v|^{2} \mathrm{~d} x+\int_{\Omega}|v|^{2(p-2)}|u-v|^{2} \mathrm{~d} x\right]^{\frac{1}{2}} \\
\leq & (p-1)\left[\left(\int_{\Omega}|u|^{2(p-2) \cdot \frac{p-1}{p-2}} \mathrm{~d} x\right)^{\frac{p-2}{p-1}}\left(\int_{\Omega}|u-v|^{2(p-1)} \mathrm{d} x\right)^{\frac{1}{p-1}}\right. \\
& \left.+\left(\int_{\Omega}|v|^{2(p-2) \cdot \frac{p-1}{p-2}} \mathrm{~d} x\right)^{\frac{p-2}{p-1}}\left(\int_{\Omega}|u-v|^{2(p-1)} \mathrm{d} x\right)^{\frac{1}{p-1}}\right]^{\frac{1}{2}} \\
= & (p-1)\left[|u|_{2(p-1)}^{2(p-2)}|u-v|_{2(p-1)}^{2}+|v|_{2(p-1)}^{2(p-2)}|u-v|_{2(p-1)}^{2}\right]^{\frac{1}{2}} \\
\leq & C\left(\|u\|^{2(p-2)}+\|v\|^{2(p-2)}\right)^{\frac{1}{2}}\|u-v\|,
\end{aligned}
$$


where $\xi(x)$ is between $u(x)$ and $v(x)$. So $f$ is locally Lipschitz continuous from $H_{0}^{2}(\Omega)$ into $L^{2}(\Omega)$.

Then, following the framework in [37, Sect. 2], by employing the theory of monotone operators and nonlinear semigroups, with energy methods, we can establish the existence of a unique local weak solution for problem (1.1).

Remark 2.4 Using the approximation technique and the contraction mapping principle, the local existence result can also be established similar to those of [10,39].

Lemma 2.5 Assume that (H1)-(H3) hold and $u$ is the weak solution of problem (1.1). Then $E(t)$ is nonincreasing and

$$
\frac{\mathrm{d}}{\mathrm{d} t} E(t)=\frac{1}{2}\left(g^{\prime} \circ \Delta u\right)(t)-\frac{1}{2} G(t)|\Delta u|_{2}^{2}-\left|u_{t}\right|_{m}^{m} \leq 0, \quad \text { a.e. } t \in\left[0, T^{*}\right) .
$$

Proof In view of the regularity of $u$ and $u_{t}$, we have $\int_{0}^{t}\left|u_{t}(\tau)\right|_{m}^{m} \mathrm{~d} \tau<\infty$,

$$
\int_{0}^{t} G(\tau)|\Delta u(\tau)|_{2}^{2} \mathrm{~d} \tau \leq \max _{\tau \in[0, t]}|\Delta u(\tau)|_{2}^{2} \int_{0}^{t} G(\tau) \mathrm{d} \tau<\infty
$$

and

$$
\begin{aligned}
\int_{0}^{t}\left(g^{\prime} \circ \Delta u\right)(\tau) \mathrm{d} \tau & =\int_{0}^{t} g^{\prime}(t-\tau)|\Delta u(t)-\Delta u(\tau)|_{2}^{2} \mathrm{~d} \tau \\
& \leq 2 \max _{\tau \in[0, t]}|\Delta u(\tau)|_{2}^{2} \int_{0}^{t} g^{\prime}(\tau) \mathrm{d} \tau<\infty .
\end{aligned}
$$

Therefore, by (2.4), $E(t)-E(0)$ is absolutely continuous on any closed subsegment of $\left[0, T^{*}\right)$. Then, by differentiating (2.4) with respect to $t$, we obtain (2.5) for a.e. $t \in\left[0, T^{*}\right)$.

By constructing proper Lyapunov's functions, Li and Gao [15] obtained the following blow-up result.

Theorem 2.6 ([15]) Assume that (H1)-(H3) hold,

$$
p>m \text { and } l>\frac{1}{(p-1)^{2}} .
$$

Then the weak solution of problem (1.1) blows up in finite time provided that the initial data $\left(u_{0}, u_{1}\right) \in H_{0}^{2}(\Omega) \times L^{2}(\Omega)$ satisfies $E(0)<E_{1}$ and $l^{\frac{1}{2}}\left|\Delta u_{0}\right|_{2}>\lambda_{1}$, where

$$
E_{1}=\left(\frac{1}{2}-\frac{1}{p}\right) \lambda_{1}^{2}, \quad \lambda_{1}=B_{1}^{-\frac{p}{p-2}}, \quad B_{1}=B l^{-\frac{1}{2}}, \quad B^{-1}=\inf _{w \in H_{0}^{2}(\Omega), w \neq 0} \frac{|\Delta w|_{2}}{|w|_{p}} .
$$

For the case of $E(0)<0$, the following blow-up result is a natural conclusion of Theorem 2.6.

Proposition 2.7 Assume that (H1)-(H3) hold,

$$
p>m \quad \text { and } l>\frac{1}{(p-1)^{2}} .
$$


Then the weak solution of problem (1.1) blows up in finite time provided that the initial data $\left(u_{0}, u_{1}\right) \in H_{0}^{2}(\Omega) \times L^{2}(\Omega)$ satisfies $E(0)<0$.

Proof For any $\left(u_{0}, u_{1}\right) \in H_{0}^{2}(\Omega) \times L^{2}(\Omega)$ such that

$$
E(0)=\frac{1}{2}\left|u_{1}\right|_{2}^{2}+\frac{1}{2}\left|\Delta u_{0}\right|_{2}^{2}-\frac{1}{p}\left|u_{0}\right|_{p}^{p}<0,
$$

it holds that

$$
0<\frac{1}{2}\left|\Delta u_{0}\right|_{2}^{2}<\frac{1}{p}\left|u_{0}\right|_{p}^{p} \leq \frac{1}{p}\left(B\left|\Delta u_{0}\right|_{2}\right)^{p} .
$$

Then

$$
\left|\Delta u_{0}\right|_{2}>\left(\frac{p}{2}\right)^{\frac{1}{p-2}} B^{-\frac{p}{p-2}}>B^{-\frac{p}{p-2}}=\left(l^{\frac{1}{2}} B_{1}\right)^{-\frac{p}{p-2}}=l^{-\frac{p}{2(p-2)}} \lambda_{1} .
$$

Since $0<l<1$, we finally obtain

$$
l^{\frac{1}{2}}\left|\Delta u_{0}\right|_{2}>l^{-\frac{1}{p-2}} \lambda_{1}>\lambda_{1} .
$$

So, by Theorem 2.6, the solution $u$ with initial data $\left(u_{0}, u_{1}\right)$ blows up in finite time.

\section{Main results}

Now, we state our main results as follows.

Theorem 3.1 Assume that (H1)-(H3) hold,

$$
p>m \quad \text { and } l>\frac{1}{(p-1)^{2}} .
$$

Then, for any $R \geq 0$, there exists a weak solution u of problem (1.1) blowing up in finite time provided that the initial data $\left(u_{0}, u_{1}\right) \in H_{0}^{2}(\Omega) \times L^{2}(\Omega)$ satisfies $E(0)=R$.

Proof In the following, we present the proof of Theorem 3.1 in three steps.

Step 1. Suppose that $u$ is a weak solution of problem (1.1) and let $\rho(t)=\int_{\Omega} u_{t} u \mathrm{~d} x$. We claim that there exist positive constants $A$ and $B$ such that

$$
\frac{\mathrm{d}}{\mathrm{d} t}(\rho(t)-B E(t)) \geq A(\rho(t)-B E(t))
$$

for all $t \in\left[0, T^{*}\right)$, where $T^{*}$ is the maximal existence time of $u$.

Because of the lack of the regularity of $u_{t t}$, the following formal calculation

$$
\frac{\mathrm{d}}{\mathrm{d} t} \rho(t)=\int_{\Omega} u_{t}^{2} \mathrm{~d} x+\int_{\Omega} u_{t t} u \mathrm{~d} x
$$

is not legitimate. Following the procedure in the proof of Theorem 1.7 in [38], we firstly verify that we can differentiate $\rho(t)$ with respect to $t$. From $(\mathrm{H} 2)$ and Theorem 2.3, it follows that

$$
u \in C\left([0, T] ; H_{0}^{2}(\Omega)\right) \subset L^{m}(\Omega \times(0, T))
$$


for any $T \in\left(0, T^{*}\right)$, thus $u \in \mathcal{F}$. Letting $\phi=u$ in (2.2), we obtain

$$
\begin{aligned}
\rho(t)= & \int_{\Omega} u_{0} u_{1} \mathrm{~d} x+\int_{0}^{t}\left|u_{t}(\tau)\right|_{2}^{2} \mathrm{~d} \tau-\int_{0}^{t}|\Delta u(\tau)|_{2}^{2} \mathrm{~d} \tau+\int_{0}^{t}|u(\tau)|_{p}^{p} \mathrm{~d} \tau \\
& -\int_{0}^{t} \int_{\Omega}\left|u_{t}(\tau)\right|^{m-2} u_{t}(\tau) u(\tau) \mathrm{d} x \mathrm{~d} \tau+\int_{0}^{t} \int_{0}^{s} g(s-\tau) \int_{\Omega} \Delta u(\tau) \Delta u(s) \mathrm{d} x \mathrm{~d} \tau \mathrm{d} s
\end{aligned}
$$

for any $t \in[0, T]$. In view of (H1)-(H3) and the regularity of $u$, for any $t \in[0, T]$, we have $\int_{0}^{t}|\Delta u(\tau)|_{2}^{2} \mathrm{~d} \tau<\infty$,

$$
\begin{aligned}
& \int_{0}^{t}\left|u_{t}(\tau)\right|_{2}^{2} \mathrm{~d} \tau \leq \int_{0}^{T}\left|u_{t}(\tau)\right|_{2}^{2} \mathrm{~d} \tau \leq T \max _{\tau \in[0, T]}\left|u_{t}(\tau)\right|_{2}^{2}<\infty \\
& \int_{0}^{t}|u(\tau)|_{p}^{p} \mathrm{~d} \tau \leq C \int_{0}^{T}|\Delta u(\tau)|_{2}^{p} \mathrm{~d} \tau \leq C T \max _{\tau \in[0, T]}|\Delta u(\tau)|_{2}^{p}<\infty \\
& \int_{0}^{t} \int_{\Omega}\left|u_{t}(\tau)\right|^{m-2} u_{t}(\tau) u(\tau) \mathrm{d} x \mathrm{~d} \tau \\
& \quad \leq \int_{0}^{T}\left|u_{t}(\tau)\right|_{m}^{m-1}|u(\tau)|_{m} \mathrm{~d} \tau \leq \frac{m-1}{m} \int_{0}^{T}\left|u_{t}(\tau)\right|_{m}^{m} \mathrm{~d} \tau+\frac{1}{m} \int_{0}^{T}|u(\tau)|_{m}^{m} \mathrm{~d} \tau<\infty \\
& \int_{0}^{t} \int_{0}^{s} g(s-\tau) \int_{\Omega} \Delta u(\tau) \Delta u(s) \mathrm{d} x \mathrm{~d} \tau \mathrm{d} s \\
& \quad \leq \frac{1}{2} \int_{0}^{T} \int_{0}^{s} g(s-\tau)\left(|\Delta u(\tau)|_{2}^{2}+|\Delta u(s)|_{2}^{2}\right) \mathrm{d} \tau \mathrm{d} s \\
& \quad \leq \max _{\tau \in[0, T]}|\Delta u(\tau)|_{2}^{2} \int_{0}^{T} \int_{0}^{s} g(s-\tau) \mathrm{d} \tau \mathrm{d} s<\infty
\end{aligned}
$$

Therefore, $\rho(t)$ is absolutely continuous on any closed subsegment of $\left[0, T^{*}\right)$. Thus, we have

$$
\begin{aligned}
\frac{\mathrm{d}}{\mathrm{d} t} \rho(t)= & \left|u_{t}\right|_{2}^{2}-|\Delta u|_{2}^{2}+|u|_{p}^{p}+\int_{0}^{t} g(t-\tau) \int_{\Omega} \Delta u(t) \Delta u(\tau) \mathrm{d} x \mathrm{~d} \tau \\
& -\int_{\Omega}\left|u_{t}\right|^{m-2} u_{t} u \mathrm{~d} x
\end{aligned}
$$

for a.e. $t \in\left[0, T^{*}\right)$. In the following, we estimate the last two terms on the right-hand side of (3.2).

Since

$$
\begin{aligned}
& \int_{0}^{t} g(t-\tau) \int_{\Omega} \Delta u(\tau) \Delta u(t) \mathrm{d} x \mathrm{~d} \tau \\
& \quad=\int_{0}^{t} g(t-\tau) \int_{\Omega}(\Delta u(\tau)-\Delta u(t)) \Delta u(t) \mathrm{d} x \mathrm{~d} \tau+\int_{0}^{t} g(t-\tau) \int_{\Omega}|\Delta u(t)|^{2} \mathrm{~d} x \mathrm{~d} \tau \\
& \quad=\int_{0}^{t} g(t-\tau) \int_{\Omega}(\Delta u(\tau)-\Delta u(t)) \Delta u(t) \mathrm{d} x \mathrm{~d} \tau+G(t)|\Delta u(t)|_{2}^{2}
\end{aligned}
$$

and

$$
\left|\int_{0}^{t} g(t-\tau) \int_{\Omega}(\Delta u(\tau)-\Delta u(t)) \Delta u(t) \mathrm{d} x \mathrm{~d} \tau\right|
$$




$$
\begin{aligned}
& \leq \int_{0}^{t} g(t-\tau)\left(\frac{1}{2} \alpha|\Delta u(t)-\Delta u(\tau)|_{2}^{2}+\frac{1}{2 \alpha}|\Delta u(t)|_{2}^{2}\right) \mathrm{d} \tau \\
& =\frac{1}{2} \alpha(g \circ \Delta u)(t)+\frac{1}{2 \alpha} G(t)|\Delta u|_{2}^{2}
\end{aligned}
$$

for any $\alpha>0$, we have

$$
\int_{0}^{t} g(t-\tau) \int_{\Omega} \Delta u(\tau) \Delta u(t) \mathrm{d} x \mathrm{~d} \tau \geq-\frac{1}{2} \alpha(g \circ \Delta u)(t)+\left(1-\frac{1}{2 \alpha}\right) G(t)|\Delta u|_{2}^{2}
$$

for any $\alpha>0$.

(i) For the case of $2<m<p$, by using Hölder's inequality and Young's inequality, we obtain

$$
\begin{aligned}
& \left.\left|\int_{\Omega}\right| u_{t}\right|^{m-2} u_{t} u \mathrm{~d} x \mid \\
& \leq \quad \int_{\Omega}\left|u_{t}\right|^{m-1}|u| \mathrm{d} x \leq\left|u_{t}\right|_{m}^{m-1}|u|_{m} \\
& \leq \leq \frac{m-1}{m}\left(\epsilon\left|u_{t}\right|_{m}^{m-1}\right)^{\frac{m}{m-1}}+\frac{1}{m}\left(\frac{1}{\epsilon}|u|_{m}\right)^{m} \\
& =\frac{m-1}{m} \epsilon^{\frac{m}{m-1}}\left|u_{t}\right|_{m}^{m}+\frac{1}{m} \frac{1}{\epsilon^{m}} \int_{\Omega}\left(\delta|u|^{\frac{p(m-2)}{p-2}}\right)\left(\frac{1}{\delta}|u|^{\frac{2(p-m)}{p-2}}\right) \mathrm{d} x \\
& \leq \quad \frac{m-1}{m} \epsilon^{\frac{m}{m-1}}\left|u_{t}\right|_{m}^{m} \\
& \quad+\frac{1}{m} \frac{1}{\epsilon^{m}} \int_{\Omega}\left[\frac{m-2}{p-2}\left(\delta|u|^{\frac{p(m-2)}{p-2}}\right)^{\frac{p-2}{m-2}}+\frac{p-m}{p-2}\left(\frac{1}{\delta}|u|^{\frac{2(p-m)}{p-2}}\right)^{\frac{p-2}{p-m}}\right] \mathrm{d} x \\
& =\frac{m-1}{m} \epsilon^{\frac{m}{m-1}}\left|u_{t}\right|_{m}^{m}+\frac{1}{m} \frac{m-2}{p-2} \frac{1}{\epsilon^{m}} \delta^{\frac{p-2}{m-2}}|u|_{p}^{p}+\frac{1}{m} \frac{p-m}{p-2} \frac{1}{\epsilon^{m}}\left(\frac{1}{\delta}\right)^{\frac{p-2}{p-m}}|u|_{2}^{2}
\end{aligned}
$$

for any $\epsilon, \delta>0$. For any $\beta>0$, there exists unique $\epsilon>0$ such that $\beta=\frac{1}{\epsilon^{m}}$. For any $\lambda>0$, the equation

$$
(m-2) \delta^{\frac{p-2}{m-2}} \lambda=(p-m)\left(\frac{1}{\delta}\right)^{\frac{p-2}{p-m}}
$$

admits a unique positive root

$$
\delta=\delta_{\lambda}:=\left(\frac{p-m}{m-2} \frac{1}{\lambda}\right)^{\frac{(m-2)(p-m)}{(p-2)^{2}}} .
$$

So, from (3.4), it follows that

$$
\begin{aligned}
\left.\left|\int_{\Omega}\right| u_{t}\right|^{m-2} u_{t} u \mathrm{~d} x \mid \leq & \frac{m-1}{m}\left(\frac{1}{\beta}\right)^{\frac{1}{m-1}}\left|u_{t}\right|_{m}^{m} \\
& +\frac{1}{m}\left(\frac{m-2}{p-2}\right)^{\frac{m-2}{p-2}}\left(\frac{1}{\lambda}\right)^{\frac{p-m}{p-2}} \beta\left(|u|_{p}^{p}+\lambda|u|_{2}^{2}\right)
\end{aligned}
$$

for any $\beta, \lambda>0$. 
By (H3) and (2.5), we have

$$
\frac{\mathrm{d}}{\mathrm{d} t} E(t) \leq-\left|u_{t}\right|_{m}^{m}
$$

Choosing arbitrary $\theta>0$, in view of (2.3) and (3.2)-(3.6), we have

$$
\begin{aligned}
& \frac{\mathrm{d}}{\mathrm{d} t}(\rho(t)-\theta E(t)) \\
& \geq \theta\left|u_{t}\right|_{m}^{m}+\left|u_{t}\right|_{2}^{2}-|\Delta u|_{2}^{2}+|u|_{p}^{p}+\left[-\frac{1}{2} \alpha(g \circ \Delta u)(t)+\left(1-\frac{1}{2 \alpha}\right) G(t)|\Delta u|_{2}^{2}\right] \\
& +\left[-\frac{m-1}{m}\left(\frac{1}{\beta}\right)^{\frac{1}{m-1}}\left|u_{t}\right|_{m}^{m}-\frac{1}{m}\left(\frac{m-2}{p-2}\right)^{\frac{m-2}{p-2}}\left(\frac{1}{\lambda}\right)^{\frac{p-m}{p-2}} \beta\left(|u|_{p}^{p}+\lambda|u|_{2}^{2}\right)\right] \\
& =\left[\left(1-\frac{1}{2 \alpha}\right) G(t)-1\right]|\Delta u|_{2}^{2}-\frac{1}{m}\left(\frac{m-2}{p-2}\right)^{\frac{m-2}{p-2}}\left(\frac{1}{\lambda}\right)^{\frac{p-m}{p-2}} \beta \lambda|u|_{2}^{2} \\
& +\left[1-\frac{1}{m}\left(\frac{m-2}{p-2}\right)^{\frac{m-2}{p-2}}\left(\frac{1}{\lambda}\right)^{\frac{p-m}{p-2}} \beta\right]|u|_{p}^{p} \\
& +\left|u_{t}\right|_{2}^{2}-\frac{1}{2} \alpha(g \circ \Delta u)(t)+\left[\theta-\frac{m-1}{m}\left(\frac{1}{\beta}\right)^{\frac{1}{m-1}}\right]\left|u_{t}\right|_{m}^{m} \\
& =\left[\left(1-\frac{1}{2 \alpha}\right) G(t)-1\right]|\Delta u|_{2}^{2}-\frac{1}{m}\left(\frac{m-2}{p-2}\right)^{\frac{m-2}{p-2}}\left(\frac{1}{\lambda}\right)^{\frac{p-m}{p-2}} \beta \lambda|u|_{2}^{2} \\
& +\left[1-\frac{1}{m}\left(\frac{m-2}{p-2}\right)^{\frac{m-2}{p-2}}\left(\frac{1}{\lambda}\right)^{\frac{p-m}{p-2}} \beta\right]|u|_{p}^{p} \\
& +\left|u_{t}\right|_{2}^{2}-\alpha\left[E(t)-\frac{1}{2}\left|u_{t}\right|_{2}^{2}-\frac{1}{2}(1-G(t))|\Delta u|_{2}^{2}+\frac{1}{p}|u|_{p}^{p}\right] \\
& +\left[\theta-\frac{m-1}{m}\left(\frac{1}{\beta}\right)^{\frac{1}{m-1}}\right]\left|u_{t}\right|_{m}^{m} \\
& =\frac{1}{2 \alpha}\left[(\alpha-1)^{2}(1-G(t))-1\right]|\Delta u|_{2}^{2}-\frac{1}{m}\left(\frac{m-2}{p-2}\right)^{\frac{m-2}{p-2}}\left(\frac{1}{\lambda}\right)^{\frac{p-m}{p-2}} \beta \lambda|u|_{2}^{2} \\
& +\left[1-\frac{1}{m}\left(\frac{m-2}{p-2}\right)^{\frac{m-2}{p-2}}\left(\frac{1}{\lambda}\right)^{\frac{p-m}{p-2}} \beta-\frac{\alpha}{p}\right]|u|_{p}^{p} \\
& +\left(1+\frac{1}{2} \alpha\right)\left|u_{t}\right|_{2}^{2}-\alpha E(t)+\left[\theta-\frac{m-1}{m}\left(\frac{1}{\beta}\right)^{\frac{1}{m-1}}\right]\left|u_{t}\right|_{m}^{m}
\end{aligned}
$$

for any $\alpha, \beta, \lambda>0$.

For any $\lambda>0$ and $0<\alpha<p$, we have

$$
\beta=\beta(\alpha, \lambda):=\left(1-\frac{\alpha}{p}\right)\left(\frac{p-2}{m-2}\right)^{\frac{m-2}{p-2}} \lambda^{\frac{p-m}{p-2}} m>0 .
$$

Since $l>\frac{1}{(p-1)^{2}}$, it holds that $1+\frac{1}{\sqrt{l}}<p$. Letting $\beta=\beta(\alpha, \lambda)$ and $\theta=\frac{m-1}{m}\left(\frac{1}{\beta(\alpha, \lambda)}\right)^{\frac{1}{m-1}}$ in $(3.7)$ and denoting

$$
\Lambda_{1}:=\inf _{\substack{w \in H_{0}^{2}(\Omega) \\ w \neq 0}} \frac{|\Delta w|_{2}^{2}}{|w|_{2}^{2}}>0
$$


we have

$$
\begin{aligned}
& \frac{\mathrm{d}}{\mathrm{d} t}\left(\rho(t)-\frac{m-1}{m}\left(\frac{1}{\beta(\alpha, \lambda)}\right)^{\frac{1}{m-1}} E(t)\right) \\
& \quad \geq \frac{1}{2 \alpha}\left[(\alpha-1)^{2}(1-G(t))-1\right]|\Delta u|_{2}^{2}-\left(1-\frac{\alpha}{p}\right) \lambda|u|_{2}^{2}+\left(1+\frac{1}{2} \alpha\right)\left|u_{t}\right|_{2}^{2}-\alpha E(t) \\
& \quad \geq \frac{1}{2 \alpha}\left[(\alpha-1)^{2} l-1\right]|\Delta u|_{2}^{2}-\left(1-\frac{\alpha}{p}\right) \lambda|u|_{2}^{2}+\left(1+\frac{1}{2} \alpha\right)\left|u_{t}\right|_{2}^{2}-\alpha E(t) \\
& \quad \geq\left[\frac{1}{2 \alpha}\left((\alpha-1)^{2} l-1\right) \Lambda_{1}-\left(1-\frac{\alpha}{p}\right) \lambda\right]|u|_{2}^{2}+\left(1+\frac{1}{2} \alpha\right)\left|u_{t}\right|_{2}^{2}-\alpha E(t)
\end{aligned}
$$

for any $\alpha \in\left(1+\frac{1}{\sqrt{l}}, p\right)$ and $\lambda>0$. Since the mapping $\alpha \mapsto \frac{1}{2 \alpha}\left[(\alpha-1)^{2} l-1\right] \Lambda_{1}$ is strictly increasing on $\left(1+\frac{1}{\sqrt{l}}, p\right)$ with

$$
\frac{1}{2 \alpha}\left[(\alpha-1)^{2} l-1\right] \Lambda_{1} \rightarrow 0^{+} \quad \text { as } \alpha \rightarrow\left(1+\frac{1}{\sqrt{l}}\right)^{+}
$$

and the mapping $\alpha \rightarrow\left(1-\frac{\alpha}{p}\right) \lambda$ is strictly decreasing on $\left(1+\frac{1}{\sqrt{l}}, p\right)$ with

$$
\left(1-\frac{\alpha}{p}\right) \lambda \rightarrow 0^{+} \quad \text { as } \alpha \rightarrow p^{-}
$$

it is easy to verify that, for any fixing $\lambda>0$, the equation

$$
\frac{1}{2 \alpha}\left[(\alpha-1)^{2} l-1\right] \Lambda_{1}=\left(1-\frac{\alpha}{p}\right) \lambda
$$

admits a unique root $\alpha(\lambda) \in\left(1+\frac{1}{\sqrt{l}}, p\right)$. Moreover, we have

$$
C(\alpha, \lambda):=\frac{1}{2 \alpha}\left[(\alpha-1)^{2} l-1\right] \Lambda_{1}-\left(1-\frac{\alpha}{p}\right) \lambda>0 \quad \text { for all } \alpha \in(\alpha(\lambda), p) .
$$

So, from (3.8), it follows that

$$
\begin{aligned}
& \frac{\mathrm{d}}{\mathrm{d} t}\left(\rho(t)-\frac{m-1}{m}\left(\frac{1}{\beta(\alpha, \lambda)}\right)^{\frac{1}{m-1}} E(t)\right) \\
& \quad \geq C(\alpha, \lambda)|u|_{2}^{2}+\left(1+\frac{1}{2} \alpha\right)\left|u_{t}\right|_{2}^{2}-\alpha E(t) \\
& \quad \geq 2 \sqrt{\left(1+\frac{1}{2} \alpha\right) C(\alpha, \lambda) \int_{\Omega} u_{t} u \mathrm{~d} x-\alpha E(t)} \\
& \quad \geq A(\alpha, \lambda)(\rho(t)-B(\alpha, \lambda) E(t))
\end{aligned}
$$

for any $\lambda>0$ and $\alpha \in(\alpha(\lambda), p)$, where

$$
A(\alpha, \lambda):=2 \sqrt{\left(1+\frac{1}{2} \alpha\right) C(\alpha, \lambda)}>0, \quad B(\alpha, \lambda):=\frac{\alpha}{A(\alpha, \lambda)}>0 .
$$


For any fixed $\lambda>0$, it is easy to verify that the mapping

$$
\alpha \mapsto \frac{m-1}{m}\left(\frac{1}{\beta(\alpha, \lambda)}\right)^{\frac{1}{m-1}}
$$

is strictly increasing on $(\alpha(\lambda), p)$ with

$$
\frac{m-1}{m}\left(\frac{1}{\beta(\alpha, \lambda)}\right)^{\frac{1}{m-1}} \rightarrow+\infty \quad \text { as } \alpha \rightarrow p^{-}
$$

Since $p>2,0<l<1$ and

$$
\begin{aligned}
B(\alpha, \lambda) & =\frac{\alpha}{A(\alpha, \lambda)} \\
& =\frac{\alpha}{2 \sqrt{\left(1+\frac{1}{2} \alpha\right) C(\alpha, \lambda)}} \\
& =\frac{\alpha}{2 \sqrt{\left(1+\frac{1}{2} \alpha\right)\left[\frac{1}{2 \alpha}\left((\alpha-1)^{2} l-1\right) \Lambda_{1}-\left(1-\frac{\alpha}{p}\right) \lambda\right]}} \\
& =\frac{\alpha}{2 \sqrt{\frac{1}{2}\left(\frac{l \Lambda_{1}}{2}+\frac{\lambda}{p}\right) \alpha^{2}-\left(\frac{\lambda}{2}-\frac{\lambda}{p}\right) \alpha-\left(\lambda+\frac{1}{4}(1+3 l) \Lambda_{1}\right)-\frac{\Lambda_{1}(1-l)}{2} \frac{1}{\alpha}}} \\
& =\frac{1}{2 \sqrt{\frac{1}{2}\left(\frac{l \Lambda_{1}}{2}+\frac{\lambda}{p}\right)-\left(\frac{\lambda}{2}-\frac{\lambda}{p}\right) \frac{1}{\alpha}-\left(\lambda+\frac{1}{4}(1+3 l) \Lambda_{1}\right) \frac{1}{\alpha^{2}}-\frac{\Lambda_{1}(1-l)}{2} \frac{1}{\alpha^{2}}}},
\end{aligned}
$$

it is easy to verify that the mapping $\alpha \mapsto B(\alpha, \lambda)$ is strictly decreasing on $(\alpha(\lambda), p)$ with $B(\alpha, \lambda) \rightarrow+\infty$ as $\alpha \rightarrow(\alpha(\lambda))^{+}$. So, for fixed $\lambda>0$, the equation

$$
B(\alpha, \lambda)=\frac{m-1}{m}\left(\frac{1}{\beta(\alpha, \lambda)}\right)^{\frac{1}{m-1}}
$$

for $\alpha$ on $(\alpha(\lambda), p)$ admits a unique root $\alpha_{0}(\lambda)$. From (3.9), it follows that

$$
\frac{\mathrm{d}}{\mathrm{d} t}\left(\rho(t)-B\left(\alpha_{0}(\lambda), \lambda\right) E(t)\right) \geq A\left(\alpha_{0}(\lambda), \lambda\right)\left(\rho(t)-B\left(\alpha_{0}(\lambda), \lambda\right) E(t)\right)
$$

for any $\lambda>0$ and $t \in\left[0, T^{*}\right)$. Choosing a fixed $\lambda>0$ and letting $A=A\left(\alpha_{0}(\lambda), \lambda\right)$ and $B=$ $B\left(\alpha_{0}(\lambda), \lambda\right)$, we get (3.1).

(ii) For the case of $m=2$, by (3.2), (3.3), and (3.6), we have

$$
\begin{aligned}
& \frac{\mathrm{d}}{\mathrm{d} t}(\rho(t)-\theta E(t)) \\
& \quad \geq\left|u_{t}\right|_{2}^{2}-|\Delta u|_{2}^{2}+|u|_{p}^{p}+\left[\left(1-\frac{1}{2 \alpha}\right) G(t)|\Delta u|_{2}^{2}-\frac{1}{2} \alpha(g \circ \Delta u)(t)\right]-\rho(t)+\theta\left|u_{t}\right|_{2}^{2} \\
& =(1+\theta)\left|u_{t}\right|_{2}^{2}+\left[\left(1-\frac{1}{2 \alpha}\right) G(t)-1\right]|\Delta u|_{2}^{2}+|u|_{p}^{p}-\rho(t) \\
& \quad-\alpha\left[E(t)-\frac{1}{2}\left|u_{t}\right|_{2}^{2}-\frac{1}{2}(1-G(t))|\Delta u|_{2}^{2}+\frac{1}{p}|u|_{p}^{p}\right]
\end{aligned}
$$




$$
\begin{aligned}
= & \left(1+\theta+\frac{1}{2} \alpha\right)\left|u_{t}\right|_{2}^{2}+\frac{1}{2 \alpha}\left[(\alpha-1)^{2}(1-G(t))-1\right]|\Delta u|_{2}^{2} \\
& +\left(1-\frac{\alpha}{p}\right)|u|_{p}^{p}-\rho(t)-\alpha E(t)
\end{aligned}
$$

for any $\alpha, \theta>0$. Since $l>\frac{1}{(p-1)^{2}}$, by letting $\alpha=p$ in (3.11), we have

$$
\frac{1}{2 p}\left[(p-1)^{2}(1-G(t))-1\right] \geq \frac{1}{2 p}\left[(p-1)^{2} l-1\right]>0 .
$$

Then, from (3.11), it follows that

$$
\begin{aligned}
& \frac{\mathrm{d}}{\mathrm{d} t}(\rho(t)-\theta E(t)) \\
& \quad=\left(1+\theta+\frac{p}{2}\right)\left|u_{t}\right|_{2}^{2}+\frac{1}{2 p}\left[(p-1)^{2}(1-G(t))-1\right]|\Delta u|_{2}^{2}-\rho(t)-p E(t) \\
& \quad \geq\left(1+\theta+\frac{p}{2}\right)\left|u_{t}\right|_{2}^{2}+\frac{1}{2 p}\left((p-1)^{2} l-1\right) \Lambda_{1}|u|_{2}^{2}-\rho(t)-p E(t) \\
& \quad \geq\left(2 \sqrt{\frac{\Lambda_{1}}{2 p}\left((p-1)^{2} l-1\right)\left(1+\frac{p}{2}+\theta\right)}-1\right) \rho(t)-p E(t) \\
& \quad=A(\theta)(\rho(t)-B(\theta) E(t))
\end{aligned}
$$

for any $\theta>\theta_{1}$, where

$$
\begin{aligned}
& \theta_{1}:=\max \left\{0, \frac{p}{2 \Lambda_{1}\left[(p-1)^{2}-1\right]}-\frac{p}{2}-1\right\}, \\
& A(\theta):=2 \sqrt{\frac{\Lambda_{1}}{2 p}\left((p-1)^{2} l-1\right)\left(1+\frac{p}{2}+\theta\right)}-1, \quad B(\theta):=\frac{p}{A(\theta)} .
\end{aligned}
$$

It is easy to verify that the equation

$$
\theta=B(\theta)=\frac{p}{2 \sqrt{\frac{\Lambda_{1}}{2 p}\left((p-1)^{2} l-1\right)\left(1+\frac{p}{2}+\theta\right)}-1}
$$

for $\theta$ on $\left(\theta_{1},+\infty\right)$ admits a unique positive $\operatorname{root} \theta_{0}$. By (3.12), we obtain

$$
\frac{\mathrm{d}}{\mathrm{d} t}\left(\rho(t)-\theta_{0} E(t)\right) \geq A\left(\theta_{0}\right)\left(\rho(t)-\theta_{0} E(t)\right)
$$

for all $t \in\left[0, T^{*}\right)$. Letting $A=A\left(\theta_{0}\right)$ and $B=\theta_{0}$, we get (3.1).

Step 2. We claim that the solution $u$ of problem (1.1) blows up in finite time provided that the initial data $\left(u_{0}, u_{1}\right) \in H_{0}^{2}(\Omega) \times L^{2}(\Omega)$ satisfies

$$
0 \leq E(0)<\frac{1}{B} \rho(0)
$$

Suppose, on the contrary, that $u$ is global. 
On the one hand, since $m \geq 2$ and $\Omega$ is bounded, there exists a positive constant $S$ depending on $m$ and $|\Omega|$ such that $|w|_{2} \leq S|w|_{m}$ for all $w \in L^{m}(\Omega)$. Then, by Theorem 2.3, (2.4) and using Hölder's inequality, we obtain

$$
\begin{aligned}
|u(t)|_{2} & =\left|u(0)+\int_{0}^{t} u_{t}(\tau) \mathrm{d} \tau\right|_{2} \\
& \leq\left|u_{0}\right|_{2}+\int_{0}^{t}\left|u_{t}(\tau)\right|_{2} \mathrm{~d} \tau \\
& \leq\left|u_{0}\right|_{2}+S \int_{0}^{t}\left|u_{t}(\tau)\right|_{m} \mathrm{~d} \tau \\
& \leq\left|u_{0}\right|_{2}+S\left(\int_{0}^{t} 1 \frac{m}{\frac{m}{m-1}} \mathrm{~d} \tau\right)^{\frac{m-1}{m}}\left(\int_{0}^{t}\left|u_{t}(\tau)\right|_{m}^{m} \mathrm{~d} \tau\right)^{\frac{1}{m}} \\
& \leq\left|u_{0}\right|_{2}+S t^{\frac{m-1}{m}}(E(0)-E(t))^{\frac{1}{m}} \quad \text { for all } t \in[0,+\infty) .
\end{aligned}
$$

Since $u=u(t)$ is a global solution of problem (1.1), we have $E(t) \geq 0$ for all $t \in[0, \infty)$. Otherwise, there exists $t_{0} \in(0, \infty)$ such that $E\left(t_{0}\right)<0$. By Proposition 2.7, $u$ blows up in finite time, which is a contradiction. Thus, due to (2.4), we have $0 \leq E(t) \leq E(0)$. Finally, in view of (3.16) we obtain

$$
|u(t)|_{2} \leq\left|u_{0}\right|_{2}+S t^{\frac{m-1}{m}}(E(0))^{\frac{1}{m}} \quad \text { for all } t \in[0,+\infty) .
$$

On the other hand, (3.1) implies that

$$
\frac{\mathrm{d}}{\mathrm{d} t} H(t) \geq A H(t) \quad \text { for all } t \in[0,+\infty)
$$

where $H(t):=\rho(t)-B E(t)$. By using Gronwall's inequality, we obtain $H(t) \geq e^{A t} H(0)$, i.e.,

$$
\int_{\Omega} u_{t} u \mathrm{~d} x=\rho(t) \geq e^{A t} H(0)+B E(t) \quad \text { for all } t \in[0,+\infty)
$$

In view of (2.4) and Theorem 2.6, and since $u(t)$ is global, we have $0 \leq E(t) \leq E(0)$, which yields that

$$
\rho(t) \geq e^{A t} H(0) \text { for all } t \in[0,+\infty)
$$

Inequality (3.15) implies that $H(0)=\rho(0)-B E(0)>0$, so we obtain

$$
\begin{aligned}
|u(t)|_{2}^{2} & =|u(0)|_{2}^{2}+2 \int_{0}^{t} \rho(\tau) \mathrm{d} \tau \\
& \geq\left|u_{0}\right|_{2}^{2}+2 \int_{0}^{t} e^{A \tau} H(0) \mathrm{d} \tau \\
& =\left|u_{0}\right|_{2}^{2}+\frac{2}{A}\left(e^{A t}-1\right) H(0)
\end{aligned}
$$

for all $t \in[0,+\infty)$, which contradicts (3.17) for $t$ sufficiently large. So $T^{*}<+\infty$. 
Step 3. For any $R \geq 0$, we can find $\left(u_{0}, u_{1}\right) \in H_{0}^{2}(\Omega) \times L^{2}(\Omega)$ such that

$$
E(0)=R<\frac{1}{B} \rho(0)
$$

In fact, let $\Omega_{1}$ and $\Omega_{2}$ be two disjoint open subdomains of $\Omega$ such that $\operatorname{dist}\left(\Omega_{1}, \partial \Omega\right)>$ 0 and $\operatorname{dist}\left(\Omega_{2}, \partial \Omega\right)>0$, and choose an arbitrary nonzero function $w \in C_{0}^{\infty}(\Omega)$ such that supp $w \subset \Omega_{1}$. Then there exists $r_{1}>0$ such that

$$
\int_{\Omega_{1}}|r w|^{2} \mathrm{~d} x=r^{2} \int_{\Omega_{1}}|w|^{2} \mathrm{~d} x>B R \quad \text { for all } r \geq r_{1} .
$$

On the other hand, by Lemma 2.1, there exists a sequence of functions $\left\{v_{k}\right\} \subset H_{0}^{2}\left(\Omega_{2}\right)$ such that

$$
\frac{1}{2} \int_{\Omega_{2}}\left|\Delta v_{k}\right|^{2} \mathrm{~d} x+\frac{1}{2} \int_{\Omega_{2}}\left|v_{k}\right|^{2} \mathrm{~d} x-\frac{1}{p} \int_{\Omega_{2}}\left|v_{k}\right|^{p} \mathrm{~d} x \rightarrow+\infty \quad \text { as } k \rightarrow \infty .
$$

Since $p>2$, there exists $r_{2}>r_{1}$ such that the function

$$
\begin{aligned}
f(r) & :=\frac{1}{2} \int_{\Omega}|r w|^{2} \mathrm{~d} x+\frac{1}{2} \int_{\Omega}|\Delta(r w)|^{2} \mathrm{~d} x-\frac{1}{p} \int_{\Omega}|r w|^{p} \mathrm{~d} x \\
& =\left(\frac{1}{2} \int_{\Omega}|w|^{2} \mathrm{~d} x+\frac{1}{2} \int_{\Omega}|\Delta w|^{2} \mathrm{~d} x\right) r^{2}-\left(\frac{1}{p} \int_{\Omega}|w|^{p} \mathrm{~d} x\right) r^{p}
\end{aligned}
$$

is continuous and strictly increasing on $\left[r_{2},+\infty\right)$, and

$$
f(r) \rightarrow-\infty \quad \text { as } r \rightarrow+\infty .
$$

So there exist $k_{0} \in \mathbb{N}$ and $r_{0} \geq r_{2}$ both sufficiently large such that

$$
\begin{aligned}
f\left(r_{2}\right) & \leq \frac{1}{2} \int_{\Omega_{2}}\left|\Delta v_{k_{0}}\right|^{2} \mathrm{~d} x+\frac{1}{2} \int_{\Omega_{2}}\left|v_{k_{0}}\right|^{2} \mathrm{~d} x-\frac{1}{p} \int_{\Omega_{2}}\left|v_{k_{0}}\right|^{p} \mathrm{~d} x=f\left(r_{0}\right) \\
& =R-\left(\frac{1}{2} \int_{\Omega}\left|r_{0} w\right|^{2} \mathrm{~d} x+\frac{1}{2} \int_{\Omega}\left|\Delta\left(r_{0} w\right)\right|^{2} \mathrm{~d} x-\frac{1}{p} \int_{\Omega}\left|r_{0} w\right|^{p} \mathrm{~d} x\right) .
\end{aligned}
$$

Let $u_{1}=u_{0}=r_{0} w+\tilde{v}$, where

$$
\tilde{v}= \begin{cases}0, & x \in \Omega \backslash \Omega_{2}, \\ v_{k_{0}}, & x \in \Omega_{2} .\end{cases}
$$

Then $u_{0} \in H_{0}^{2}(\Omega), u_{1} \in L^{2}(\Omega)$. By Theorem 2.3, problem (1.1) has a unique weak solution $u$ with initial data $\left(u_{0}, u_{1}\right)$ such that

$$
u \in C\left([0, T] ; H_{0}^{2}(\Omega)\right), \quad u_{t} \in C\left([0, T] ; L^{2}(\Omega)\right) \cap L^{m}(\Omega \times(0, T))
$$

for some $T>0$. In view of (2.3), (3.18), and (3.19), we have

$$
\rho(0)=\int_{\Omega} u_{0} u_{1} \mathrm{~d} x=\int_{\Omega}\left|r_{0} w+\tilde{v}\right|^{2} \mathrm{~d} x \geq \int_{\Omega_{1}}\left|r_{0} w+\tilde{v}\right|^{2} \mathrm{~d} x=\int_{\Omega_{1}}\left|r_{0} w\right|^{2} \mathrm{~d} x>B R
$$


and

$$
\begin{aligned}
E(0)= & \frac{1}{2}\left|u_{t}(0)\right|_{2}^{2}+\frac{1}{2}(1-G(0))|\Delta u(0)|_{2}^{2}+\frac{1}{2}(g \circ \Delta u)(0)-\frac{1}{p}|u(0)|_{p}^{p} \\
= & \frac{1}{2}\left|u_{1}\right|_{2}^{2}+\frac{1}{2}\left|\Delta u_{0}\right|_{2}^{2}-\frac{1}{p}\left|u_{0}\right|_{p}^{p} \\
= & \frac{1}{2}\left(\int_{\Omega_{1}}\left|r_{0} w\right|^{2} \mathrm{~d} x+\int_{\Omega_{2}}|\tilde{v}|^{2} \mathrm{~d} x\right)+\frac{1}{2}\left(\int_{\Omega_{1}}\left|\Delta\left(r_{0} w\right)\right|^{2} \mathrm{~d} x+\int_{\Omega_{2}}|\Delta \tilde{v}|^{2} \mathrm{~d} x\right) \\
& -\frac{1}{p}\left(\int_{\Omega_{1}}\left|r_{0} w\right|^{p} \mathrm{~d} x+\int_{\Omega_{2}}|\tilde{v}|^{p} \mathrm{~d} x\right) \\
= & R<\frac{1}{B} \rho(0) .
\end{aligned}
$$

Hence, according to the conclusion in Step 2, the weak solution $u$ blows up in finite time.

This completes the proof.

In view of (3.10), $B(\alpha, \lambda)$ is strictly decreasing for $\alpha$ on $(\alpha(\lambda), p)$, then we have

$$
B_{0}:=\inf _{\lambda>0} B\left(\alpha_{0}(\lambda), \lambda\right) \geq B(p, \lambda)=\frac{p}{\sqrt{\left(1+\frac{2}{p}\right)\left((p-1)^{2} l-1\right) \Lambda_{1}}}>0 .
$$

For the case of $m=2$, we denote $B_{0}=\theta_{0}$, where $\theta_{0}$ is the unique root of equation (3.13). Then, in view of the proof of Theorem 3.1, we obtain the following result.

Corollary 3.2 Assume that (H1)-(H3) hold,

$$
p>m \text { and } l>\frac{1}{(p-1)^{2}} .
$$

Then the weak solution of problem (1.1) blows up in finite time provided that the initial data $\left(u_{0}, u_{1}\right) \in H_{0}^{2}(\Omega) \times L^{2}(\Omega)$ satisfies

$$
0 \leq E(0)<\frac{1}{B_{0}} \int_{\Omega} u_{0} u_{1} \mathrm{~d} x
$$

Proof We only need to consider the case of $2<m<p$. Noticing that the mapping $\lambda \mapsto$ $B\left(\alpha_{0}(\lambda), \lambda\right)$ is continuous on $(0,+\infty)$, by (3.20) and (3.21), there exists $\lambda_{0}>0$ such that

$$
0 \leq E(0)<\frac{1}{B\left(\alpha_{0}\left(\lambda_{0}\right), \lambda_{0}\right)} \int_{\Omega} u_{0} u_{1} \mathrm{~d} x \leq \frac{1}{B_{0}} \int_{\Omega} u_{0} u_{1} \mathrm{~d} x
$$

Following the proof of Theorem 3.1, the weak solution $u$ blows up in finite time.

Remark 3.3 If we follow the strategy in [19] directly without the modifications, we will obtain the coefficient $B\left(\alpha_{0}(\lambda), \lambda\right)$ only for the specified parameter $\lambda=\frac{p-m}{p-2}$.

Remark 3.4 Denote

$$
\mathbb{B}_{1}:=\left\{\left(u_{0}, u_{1}\right) \in H_{0}^{2}(\Omega) \times L^{2}(\Omega): E(0)<E_{1}, l^{\frac{1}{2}}\left|\Delta u_{0}\right|>\lambda_{1}\right\}
$$


and

$$
\mathbb{B}_{2}:=\left\{\left(u_{0}, u_{1}\right) \in H_{0}^{2}(\Omega) \times L^{2}(\Omega): 0 \leq E(0)<\frac{1}{B_{0}} \int_{\Omega} u_{0} u_{1} \mathrm{~d} x\right\}
$$

In view of Theorem 2.6 and Corollary 3.2, the solution $u$ for problem (1.1) with initial data $\left(u_{0}, u_{1}\right) \in \mathbb{B}_{1} \cup \mathbb{B}_{2}$ blows up in finite time. On the one hand, for any $\left(u_{0}, u_{1}\right) \in H_{0}^{2}(\Omega) \times$ $L^{2}(\Omega)$ satisfying $E(0)<0$, Proposition 2.7 implies that $\left(u_{0}, u_{1}\right) \in \mathbb{B}_{1} \backslash \mathbb{B}_{2}$. On the other hand, Theorem 3.1 implies that $\mathbb{B}_{2} \backslash \mathbb{B}_{1} \neq \emptyset$.

\section{Acknowledgements}

The authors would like to thank the referees for their useful suggestions which have significantly improved the paper.

\section{Funding}

This work was supported financially by the National Natural Science Foundation of China (11871302), Natural Science Foundation of Shandong Province of China (ZR2017MA036).

\section{Availability of data and materials}

Data sharing not applicable to this article as no datasets were generated or analysed during the current study.

\section{Competing interests}

The authors declare that there is no conflict of interests regarding the publication of this paper.

\section{Authors' contributions}

All authors contributed equally and significantly in writing this article. All authors read and approved the final manuscript.

\section{Author details}

'School of Mathematical Sciences, Qufu Normal University, Qufu, People's Republic of China. ${ }^{2}$ Department of Mathematics and Statistics, Curtin University, Perth, Australia.

\section{Publisher's Note}

Springer Nature remains neutral with regard to jurisdictional claims in published maps and institutional affiliations.

Received: 20 June 2018 Accepted: 16 January 2019 Published online: 22 January 2019

\section{References}

1. Han, X., Wang, M.: Asymptotic behavior for Petrovsky equation with localized damping. Acta Appl. Math. 110(3), 1057-1076 (2010). https://doi.org/10.1007/s10440-009-9493-6

2. Li, G., Sun, Y., Liu, W.: Global existence, uniform decay and blow-up of solutions for a system of Petrovsky equations. Nonlinear Anal. 74(4), 1523-1538 (2011). https://doi.org/10.1016/j.na.2010.10.025

3. Li, G., Sun, Y., Liu, W.: Global existence and blow-up of solutions for a strongly damped Petrovsky system with nonlinear damping. Appl. Anal. 91(3), 575-586 (2012). https://doi.org/10.1080/00036811.2010.550576

4. Wang, Y., Wang, Y.: On the initial-boundary problem for fourth order wave equations with damping, strain and source terms. J. Math. Anal. Appl. 405(1), 116-127 (2013). https://doi.org/10.1016/j.jmaa.2013.03.060

5. Zhou, J.: Lower bounds for blow-up time of two nonlinear wave equations. Appl. Math. Lett. 45, 64-68 (2015). https://doi.org/10.1016/j.aml.2015.01.010

6. Zhou, J.: Global existence and blow-up of solutions for a Kirchhoff type plate equation with damping. Appl. Math. Comput. 265, 807-818 (2015). https://doi.org/10.1016/j.amc.2015.05.098

7. Ye, Y.: Global existence and blow-up of solutions for a system of Petrovsky equations. Appl. Anal. 96(16), 2869-2890 (2017). https://doi.org/10.1080/00036811.2016.1249862

8. Al-Gharabli, M.M., Messaoudi, S.A.: The existence and the asymptotic behavior of a plate equation with frictional damping and a logarithmic source term. J. Math. Anal. Appl. 454(2), 1114-1128 (2017). https://doi.org/10.1016/j.jmaa.2017.05.030

9. Mustafa, M.I., Kafini, M.: Decay rates for memory-type plate system with delay and source term. Math. Methods Appl. Sci. 40(4), 883-895 (2017). https://doi.org/10.1002/mma.4015

10. Messaoudi, S.A.: Global existence and nonexistence in a system of Petrovsky. J. Math. Anal. Appl. 265(2), 296-308 (2002). https://doi.org/10.1006/jmaa.2001.7697

11. Messaoudi, S.A.: Blow up and global existence in a nonlinear viscoelastic wave equation. Math. Nachr. 260(1), 58-66 (2003). https://doi.org/10.1002/mana.200310104

12. Georgiev, V., Todorova, G.: Existence of a solution of the wave equation with nonlinear damping and source terms. J. Differ. Equ. 109(2), 295-308 (1994). https://doi.org/10.1006/jdeq.1994.1051

13. Messaoudi, S.A.: Blow-up of positive-initial-energy solutions of a nonlinear viscoelastic hyperbolic equation. J. Math. Anal. Appl. 320(2), 902-915 (2006). https://doi.org/10.1016/j.jmaa.2005.07.022

14. Chen, W., Zhou, Y.: Global nonexistence for a semilinear Petrovsky equation. Nonlinear Anal. 70(9), 3203-3208 (2009). https://doi.org/10.1016/j.na.2008.04.024 
15. Li, F., Gao, Q.: Blow-up of solution for a nonlinear Petrovsky type equation with memory. Appl. Math. Comput. 274, 383-392 (2016). https://doi.org/10.1016/j.amc.2015.11.018

16. Todorova, G., Vitillaro, E.: Blow-up for nonlinear dissipative wave equations in $\mathbb{R}^{n}$. J. Math. Anal. Appl. 303(1), 242-257 (2005). https://doi.org/10.1016/j.jmaa.2004.08.039

17. Ye, Y: Global existence and blow-up of solutions for higher-order viscoelastic wave equation with a nonlinear source term. Nonlinear Anal. 112, 129-146 (2015). https://doi.org/10.1016/i.na.2014.09.001

18. Song, H., Xue, D.: Blow up in a nonlinear viscoelastic wave equation with strong damping. Nonlinear Anal. 109, 245-251 (2014). https://doi.org/10.1016/..na.2014.06.012

19. Song, H.: Blow up of arbitrarily positive initial energy solutions for a viscoelastic wave equation. Nonlinear Anal., Real World Appl. 26, 306-314 (2015). https://doi.org/10.1016/j.nonrwa.2015.05.015

20. Sun, F., Liu, L., Wu, Y.: Finite time blow-up for a thin-film equation with initial data at arbitrary energy level. J. Math. Anal. Appl. 458(1), 9-20 (2018). https://doi.org/10.1016/j.jmaa.2017.08.047

21. Sun, F., Liu, L., Wu, Y.: Global existence and finite time blow-up of solutions for the semilinear pseudo-parabolic equation with a memory term. Appl. Anal., 1-21 (2017). https://doi.org/10.1080/00036811.2017.1400536

22. Sun, F., Liu, L., Wu, Y.: Finite time blow-up for a class of parabolic or pseudo-parabolic equations. Comput. Math. Appl. 75(10), 3685-3701 (2018). https://doi.org/10.1016/j.camwa.2018.02.025

23. Sun, F., Liu, L., Wu, Y.: Blow-up of solutions for a nonlinear viscoelastic wave equation with initial data at arbitrary energy level. Appl. Anal., 1-20 (2018). https://doi.org/10.1080/00036811.2018.1460812

24. Gazzola, F., Squassina, M.: Global solutions and finite time blow up for damped semilinear wave equations. Ann. Inst. Henri Poincaré, Anal. Non Linéaire 23(2), 185-207 (2006). https://doi.org/10.1016/j.anihpc.2005.02.007

25. Wang, Y:: A global nonexistence theorem for viscoelastic equations with arbitrary positive initial energy. Appl. Math Lett. 22(9), 1394-1400 (2009). https://doi.org/10.1016/j.aml.2009.01.052

26. Zeng, R., Mu, C., Zhou, S.: A blow-up result for Kirchhoff-type equations with high energy. Math. Methods Appl. Sci. 34(4). 479-486 (2011). https://doi.org/10.1002/mma.1374

27. Korpusov, M.O.: Blow-up of the solution of a nonlinear system of equations with positive energy. Theor. Math. Phys. 171(3), 725-738 (2012). https://doi.org/10.1007/s11232-012-0070-1

28. Kutev, N., Kolkovska, N., Dimova, M.: Sign-preserving functionals and blow-up to Klein-Gordon equation with arbitrary high energy. Appl. Anal. 95(4), 860-873 (2016). https://doi.org/10.1080/00036811.2015.1038994

29. Zhu, X., Li, F., Rong, T.: Global existence and blow up of solutions to a class of pseudo-parabolic equations with an exponential source. Commun. Pure Appl. Anal. 14(6), 2465-2485 (2015). https://doi.org/10.3934/cpaa.2015.14.2465

30. Zhu, X., Li, F., Li, Y.: A new sufficient condition for blow-up of solutions to a class of parabolic equations. Appl. Anal. 95(5), 1137-1143 (2016). https://doi.org/10.1080/00036811.2015.1053052

31. Zhu, X., Li, F., Liang, Z., Rong, T.: A sufficient condition for blowup of solutions to a class of pseudo-parabolic equations with a nonlocal term. Math. Methods Appl. Sci. 39(13), 3591-3606 (2016). https://doi.org/10.1002/mma.3803

32. Zhu, X. Li, F., Li, Y.: Some sharp results about the global existence and blowup of solutions to a class of pseudo-parabolic equations. Proc. R. Soc. Edinb. 147A, 1-21 (2017). https://doi.org/10.1017/S0308210516000494

33. Zhu, X., Li, F., Li, Y.: Global solutions and blow up solutions to a class of pseudo-parabolic equations with nonlocal term. Appl. Math. Comput. 329, 38-51 (2018). https://doi.org/10.1016/j.amc.2018.02.003

34. Han, Y:: A class of fourth-order parabolic equation with arbitrary initial energy. Nonlinear Anal., Real World Appl. 43 , 451-466 (2018). https://doi.org/10.1016/j.nonrwa.2018.03.009

35. Han, Y., Li, Q:: Threshold results for the existence of global and blow-up solutions to Kirchhoff equations with arbitrary initial energy. Comput. Math. Appl. 75(9), 3283-3297 (2018). https://doi.org/10.1016/j.camwa.2018.01.047

36. Willem, M.: Minimax Theorems. Progress in Nonlinear Differential Equations and Their Applications, vol. 24. Birkhäuser Boston, Boston (1996)

37. Guo, Y., Rammaha, M.A., Sakuntasathien, S., Titi, E.S., Toundykov, D.: Hadamard well-posedness for a hyperbolic equation of viscoelasticity with supercritical sources and damping. J. Differ. Equ. 257(10), 3778-3812 (2014). https://doi.org/10.1016/j.jde.2014.07.009

38. Guo, Y., Rammaha, M.A., Sakuntasathien, S.: Blow-up of a hyperbolic equation of viscoelasticity with supercritical nonlinearities. J. Differ. Equ. 262(3), 1956-1979 (2017). https://doi.org/10.1016/j.jde.2016.10.037

39. Gao, Q., Li, F., Wang, Y.: Blow-up of the solution for higher-order Kirchhoff-type equations with nonlinear dissipation. Cent. Eur. J. Math. 9(3), 686-698 (2011). https://doi.org/10.2478/s11533-010-0096-2

\section{Submit your manuscript to a SpringerOpen ${ }^{\circ}$ journal and benefit from:}

- Convenient online submission

- Rigorous peer review

- Open access: articles freely available online

- High visibility within the field

- Retaining the copyright to your article

Submit your next manuscript at $\boldsymbol{~ s p r i n g e r o p e n . c o m ~}$ 